\title{
Demonstrating Contexta-CARE: a Situation-Aware System for Supporting Independent Living
}

\author{
Davide Merico, Roberto Bisiani, \\ Fabio Malizia and Gabriele Rizzi \\ Contexta Network Solutions S.r.l. \\ via Carducci 16, I-20123 Milan, Italy \\ davide.merico@contexta.it
}

\author{
Hashim Ali \\ University of Milan-Bicocca, DISCo, \\ viale Sarca 336/14, I-20126 Milan, Italy \\ hashim.ali@disco.unimib.it
}

\begin{abstract}
In this paper we propose the demonstration of an Independent Living system that exploits an approach based on situation awareness and ambient intelligence in order to improve the quality of life of users that want to live independently. This approach aims at extending "awareness" bringing to bear new ambient data and opening the possibility of "reasoning" on complex sequences of events in relation to the context they occur in, i.e. particular situations such as falls.

Contexta-CARE, the situation-aware independent-living system we implemented in order to validate the approach, enables close monitoring of a person as well as of the environment where the person lives. Contexta-CARE has the ability to both highlight, preventively, situations that might evolve into potentially dangerous events, and to promptly signal critical events, like falls. The demonstration will highlight all the details of the particular approach we are proposing.
\end{abstract}

Index Terms-Situation Recognition, Context-aware, Situation Awareness, Independent Living, Wireless Sensor Networks

\section{INTRODUCTION}

We are rapidly heading towards the realization of paradigms commonly described as ubiquitous computing [1], pervasive computing, ambient intelligence, or, more recently, "everyware" [2]. These paradigms envision living environments pervaded by a high number of visible and invisible devices affecting and improving all aspects of our lives.

With the increasing availability of deployable sensors and computer devices we are more and more capable of making environments aware of situations, from a single elderly person in distress to an earthquake endangering millions of people.

Perceiving the environment, understanding what factors are meaningful in particular situations can make a big difference on decision making. Pervasive, automatic and continuous situation awareness will be at the core of several applications and products in the field of ambient intelligence. In particular, given our past experiences [3] we believe that situation awareness can be particularly useful to improve Independent Living systems.

Therefore, we designed and realized Contexta-CARE, a situation-aware independent-living system, which enables close monitoring of a person as well as of the environment where the person lives. One of the main features of ContextaCARE is its ability to both highlight, preventively, situations that might evolve into potentially dangerous events, and to promptly signal critical events, like falls.

This paper gives a brief introduction to the system focusing on an architectural overview and on detailing a few aspects about the situation-aware approach we propose.

\section{CONTEXTA-CARE: SituAtion AWARENESS FOR INDEPENDENT LIVING}

As also shown in Figure 1, the architecture of the ContextaCARE system is based on two main components.

The first component is a Wireless Sensor Network (WSN) [4] called Contexta-NET [5] that is used for gathering data about the user and the environment.

The Contexta-NET WSN is based on the IEEE 802.15.4 protocol and it is composed of environmental and wearable sensors, connected via radio to the intelligent data concentrator capable of analyzing and storing the gathered data.

The WSN is used for collecting data to pinpoint the position and the motion activities of the user and moreover it is used to collect environmental data (e.g. ambient light, temperature, humidity, opening and closing of doors or gates). Further details about Contexta-NET can be found in [5].

The second main component is an intelligent dataconcentrator that computes high-level information about the gathered data. This component is also used for detecting and recognizing any unusual situation or emergency. Moreover, it implements a multi-sensory tracking engine, mainly used to compute the user location and movements, and a situation assessment component, used for better understanding the particular contextual data about the situation that is occurring.

Therefore, the data collected with the sensor network are used to support situation-aware components for: (i) continuously analyzing the physical activities carried out by the user; (ii) predicting possible risky situations; (iii) identifying the causes that lead to a change of state in user's health.

The result of the processing can be used in many ways, in its simplest form it can be a simple message to a caregiver (via SMS or email). More sophisticated output produced by the system can be periodically sent to specially trained nurses or physicians for detailed analysis. 


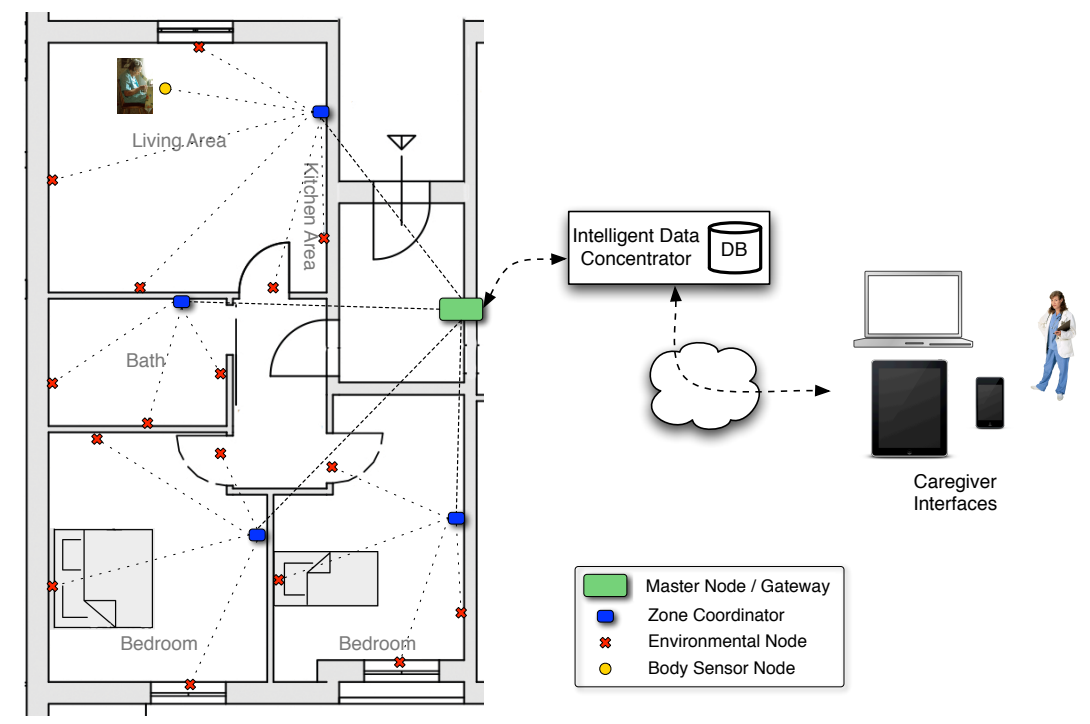

Fig. 1: The Architecture of the Contexta-CARE Independent Living System.

\section{Situation-AWARE Component}

The intelligent data-concentrator component is used for correlating all the information about the context in order to better characterize the current and the future situations. Particular situations can be modeled using knowledge from a particular field (e.g. medical knowledge) or common-sense behavior.

The goal is trying to recognize a situation, highlighting a particular sequence of events, rather than a single event. For example, the component can emphasize sequences of unnatural postures or movements, automatically adapting to the typical behavior of the user. The observation of chains of events instead of single actions drastically reduces false positives problems.

As previously hinted, this component computes two kinds of information:

- movements in a given zone and proximity, e.g. entering and exiting zones:

- situation information that merge position and context, e.g. the user is in bed, the user is in a dark room.

If we only use movement information the component can only generate a proximity information. If the context model is added [6], Contexta-CARE can compute information on what might have happened and therefore give a more precise tracking of the user. This component is driven by a number of rules that infer the occurrence of specific events and movements.

We implemented the core code of the component using a rule management system called Drools [7]. Drools is a business rule management system (BRMS) with a forwardchaining inference-based rules engine, more correctly known as a production rule system, using an enhanced implementation of the Rete [8] algorithm.

The situation-recognition rules we have implemented belong to three categories:
- Rules that use only movement features;

- Rules that use only environmental features;

- Rules that use both kinds of information.

During the demonstration we will detail these rules with usage examples in a real setting.

\section{CONCLUSION}

In this paper we proposed a demonstration of ContextaCARE, an Independent Living system that exploits situation awareness and ambient intelligence in order to improve the quality of life of users that want to live independently. Contexta-CARE is currently under test in an independentliving environment composed of four apartments with 15 users.

In the demonstration we will show the system capabilities, focusing on user the particular approach we are proposing, and showing real examples of usage.

\section{REFERENCES}

[1] M. Weiser, "The computer for the 21st century," Scientific American, February 1991. [Online]. Available: http://www.ubiq.com/hypertext/ weiser/SciAmDraft3.html

[2] A. Greenfield, Everyware: The Dawning Age of Ubiquitous Computing. New Riders, 2006.

[3] R. Bisiani, D. Merico, A. Mileo, and S. Pinardi, "A logical approach to home healthcare with intelligent sensor-network support," The Computer Journal, p. bxn074, 2009.

[4] I. F. Akyildiz, W. Su, Y. Sankarasubramaniam, and E. Cayirci, "A survey on sensor networks," IEEE Communications Magazine, vol. 40, no. 8, pp. 102-114, 2002.

[5] D. Merico, R. Bisiani, and F. Malizia, "Localization with ambient sensors," in Indoor Positioning and Indoor Navigation (IPIN), 2011 International Conference on, sept. 2011, pp. 21-23.

[6] A. Mileo, D. Merico, and R. Bisiani, "Support for context-aware monitoring in home healthcare," Journal of Ambient Intelligence and Smart Environments, vol. 2, no. 1, pp. 49-66, 01 2010/01/01/. [Online]. Available: http://dx.doi.org/10.3233/AIS-2010-0052

[7] P. Browne, JBoss Drools Business Rules. Packt Publishing, 2009.

[8] C. L. Forgy, "Rete: A fast algorithm for the many pattern/many object pattern match problem," Artificial Intelligence, vol. 19, no. 1, pp. 17-37, 1982. 